\title{
A Case of Myelodysplastic Syndrome with Clinical Manifestations of Recurrent Cellulitis
}

\author{
Jung Han Lim ${ }^{1}$, Sue Min Kim² (D), Seungyun Jee ${ }^{3}$, Seong Oh Park ${ }^{1}$ (1) \\ ${ }^{1}$ Department of Plastic and Reconstructive Surgery, Hanyang University College of Medicine, Seoul; ${ }^{2}$ Department of Plastic and Reconstructive Surgery, \\ National Medical Center, Seoul; ${ }^{3}$ Department of Pathology, Hanyang University College of Medicine, Seoul, Korea
}

\begin{abstract}
An 84-year-old male patient with recurrent cellulitis in the left submandibular area visited the outpatient clinic. He underwent debridement of the wound with administration of intravenous antibiotics. The defect was reconstructed using a split-thickness skin graft. After 2 months, the cellulitis recurred adjacent to the prior lesion in the submandibular area. The wound improved with intravenous antibiotics and conservative treatment. During the second hospitalization, the patient was evaluated for abnormal complete blood count, and was finally diagnosed with myelodysplastic syndromes. Recurrent cellulitis in this patient was not accompanied with any other symptoms; instead of being a simple uncontrolled infection it had a systemic origin. Physicians should consider the possibility of systemic causes for recurrent cellulitis, such as myelodysplastic syndromes, especially if laboratory results suggest pancytopenia.
\end{abstract}

Keywords: Myelodysplastic syndrome; Recurrent cellulitis; Cellulitis; Pancytopenia

\section{Introduction}

Myelodysplastic syndromes (MDS) are caused by malignant hematopoietic stem cells [1]. Though peripheral blood cytopenia with dysplastic changes in hypercellular bone marrow are hallmarks of MDS, its clinical manifestations are not distinctive. Some patients may present with variable symptoms including chronic fatigue, petechiae, infections, and other complications of cytopenia, while others may be asymptomatic $[2,3]$. Herein, we present a case of unexplained recurrent cellulitis with no other specific symptoms; the patient was finally diagnosed with MDS during his second hospital stay for treatment of recurrent cellulitis. Informed consent for use of photographs was obtained from the patient.

\section{Case}

An 84-year-old male patient was referred for recurrent swelling and pus-like discharge over the left side of his neck for 2 weeks prior to presentation. The patient had been diagnosed with cellulitis and abscess formation and had undergone repeated incision and drainage at another facility before visiting us. The initial physical examination revealed a $5 \times 3 \mathrm{~cm}$, severely painful, suppurative ulcer in the left submandibular region (Fig. 1A). Enhanced computed tomography (CT) showed an enhanced skin thickening $6 \mathrm{~cm}$ in diameter with central necrosis in the left submandibular area, implying cellulitis or malignancy of the skin. There was no visible abscess formation or evidence of cervical lymphadenopathy (Fig. 1B). The patient underwent debridement

\section{Case Report}

Received: September 15, 2020

Revised: October 15, 2020

Accepted: October 21, 2020

\section{Corresponding author: Seong Oh Park, M.D., M.S.}

Department of Plastic and Reconstructive Surgery, Hanyang University College of Medicine, 222-1 Wangsimni-ro, Seongdonggu, Seoul 04763, Korea

Tel: +82-2-2290-8567

Fax: +82-2-2290-8464

E-mail: psopark950@gmail.com

This is an Open Access article distributed under the terms of the Creative Commons Attribution Non-Commercia License (https://creativecommons.org/licenses/by-nc/4.O/) which permits unrestricted non-commercial use, distribution, and reproduction in any medium, provided the original work is properly cited.

(C) 2020 Korean Wound Management Society 

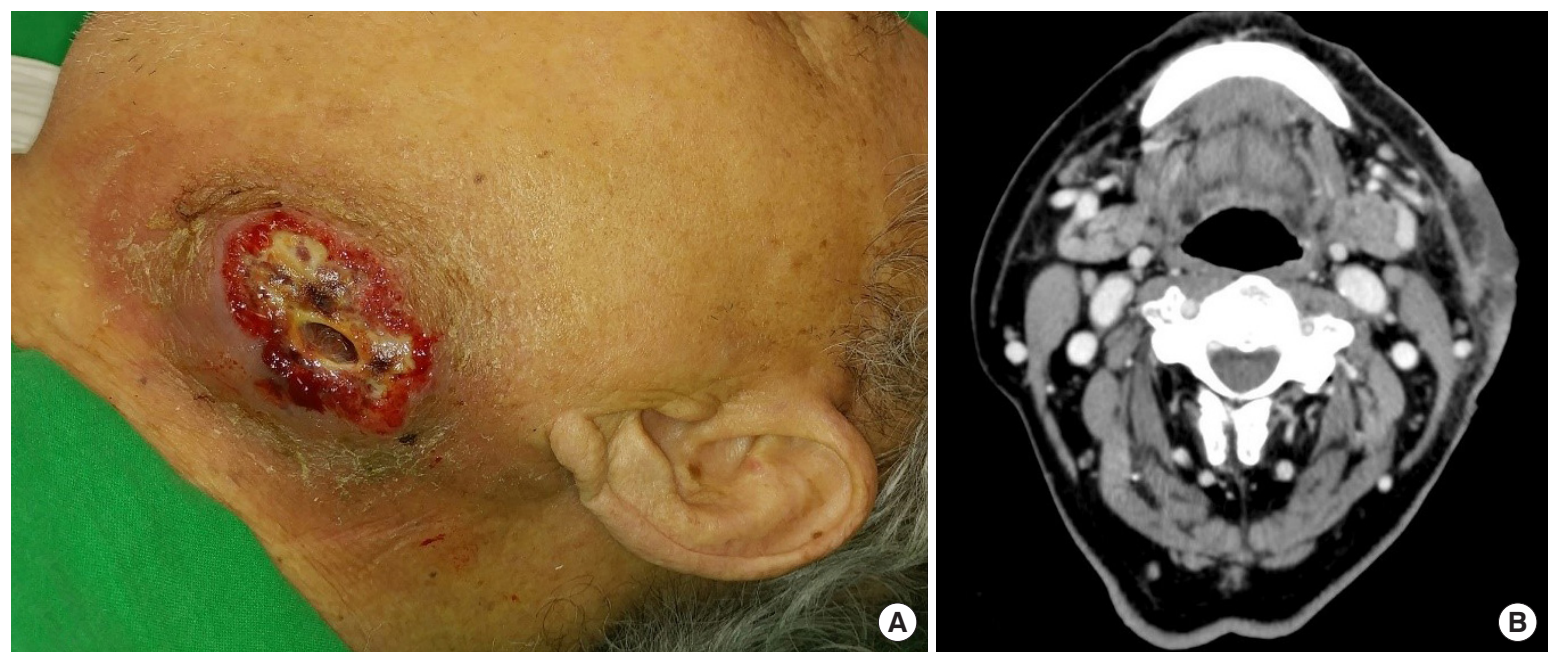

Fig. 1. The initial findings. (A) A $5 \times 3 \mathrm{~cm}$, severely painful suppurative ulcer in the left submandibular region. (B) Enhanced neck computed tomography showing enhanced skin thickening and central necrosis at the left submandibular area with fat stranding and fascia thickening at the subcutaneous layer.
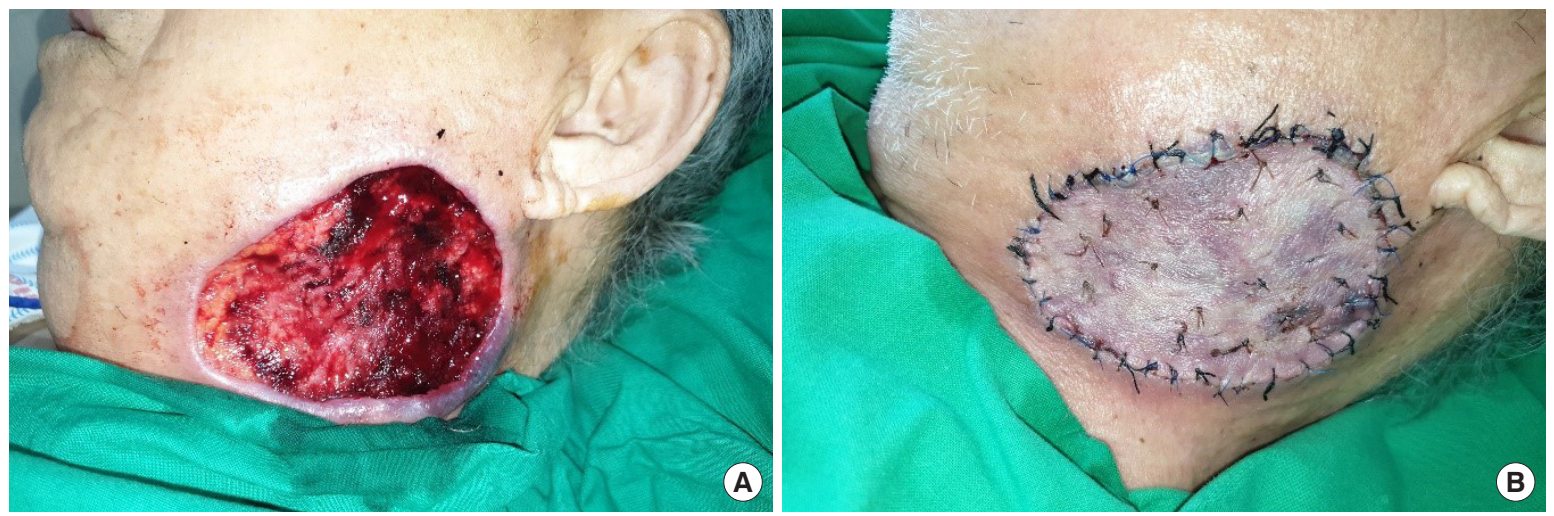

Fig. 2. Clinical course. (A) Defect after debridement and negative wound therapy. (B) Reconstruction of the defect using split-thickness skin graft.

and negative pressure wound therapy, with bacterial culture and histopathologic examinations (Fig. 2A). Initial lab findings showed results suggesting pancytopenia; his white blood cell count was $4.4 \times 10^{3} / \mathrm{mm}^{3}$, hemoglobin was $7.8 \mathrm{~g} / \mathrm{dL}$, and platelet count was $128 \times 10^{3} / \mathrm{mm}^{3}$. There was no febrile episode. Methicillin-resistant Staphylococcus aureus (MRSA) was identified in the wound culture. Histologic findings showed acute necrotizing suppurative inflammation (Fig. 3).

After administration of intravenous antibiotics, the wound improved and stabilized with decreased discharge. The defect was reconstructed using a split-thickness skin graft (Fig. 2B). During hospitalization, the patient was referred to the hematooncology department for evaluation of pancytopenia. On gastrofiberscopy, there was no evidence of gastrointestinal bleeding. The patient's neutropenia was believed to be drug-induced, possibly due to antibiotics. The patient was managed conservatively and discharged with a completely healed wound.

Two months later, the patient returned with a localized inflammation on the neck adjacent to where he had the skin graft. He presented with pain similar to the past episode, ulcerative lesion, and fever (Fig. 4A and B). The initial laboratory findings showed pancytopenia and elevated C-reactive protein (CRP) (white blood cell, $4.4 \times 10^{3} / \mu \mathrm{L}$; hemoglobin, $9.6 \mathrm{~g} / \mathrm{dL}$; platelet, $128 \times 10^{3} / \mu \mathrm{L}$; CRP, $3.51 \mathrm{mg} / \mathrm{dL}$ ). Enhanced neck CT showed findings suggestive of cellulitis at the left posterior neck. Histopathologic findings demonstrated acute suppurative inflammation, and MRSA was cultured again. After administration of intravenous vancomycin to target the MRSA, the ulcerative lesion improved. However, the follow-up laboratory findings showed aggravated pancytopenia and the patient 

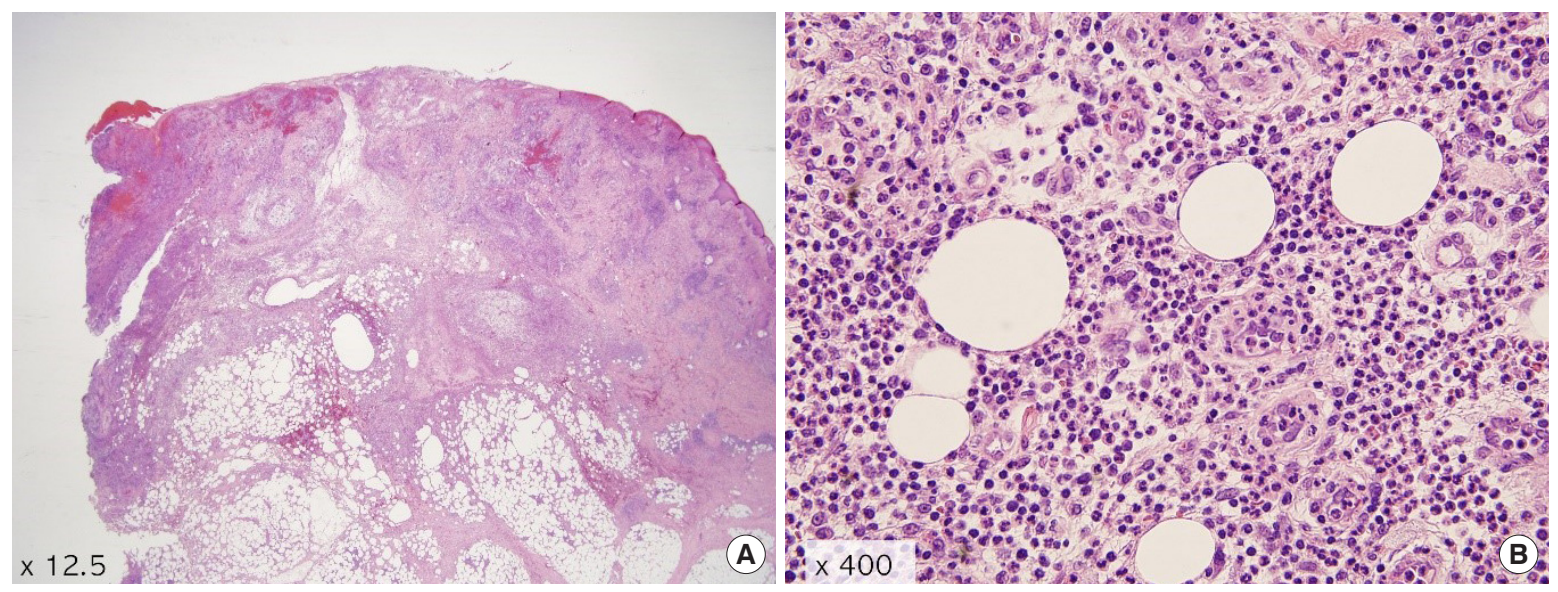

Fig. 3. Soft tissue biopsy findings. (A, B) Acute necrotizing suppurative inflammation with complete denudation of epidermis, and lobular and septal panniculitis (hematoxylin and eosin stain).
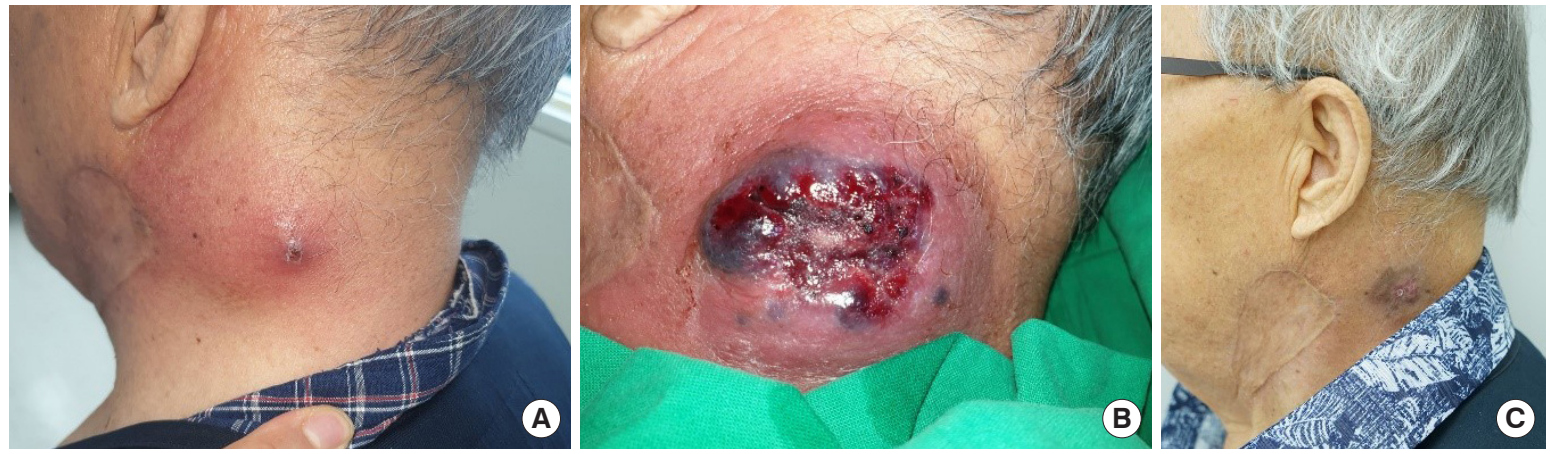

Fig. 4. Clinical course during the second hospital stay. (A) Recurred skin lesion adjacent to prior split-thickness skin graft site. (B) Aggravated skin lesion during hospital stay. (C) Completely healed wound.
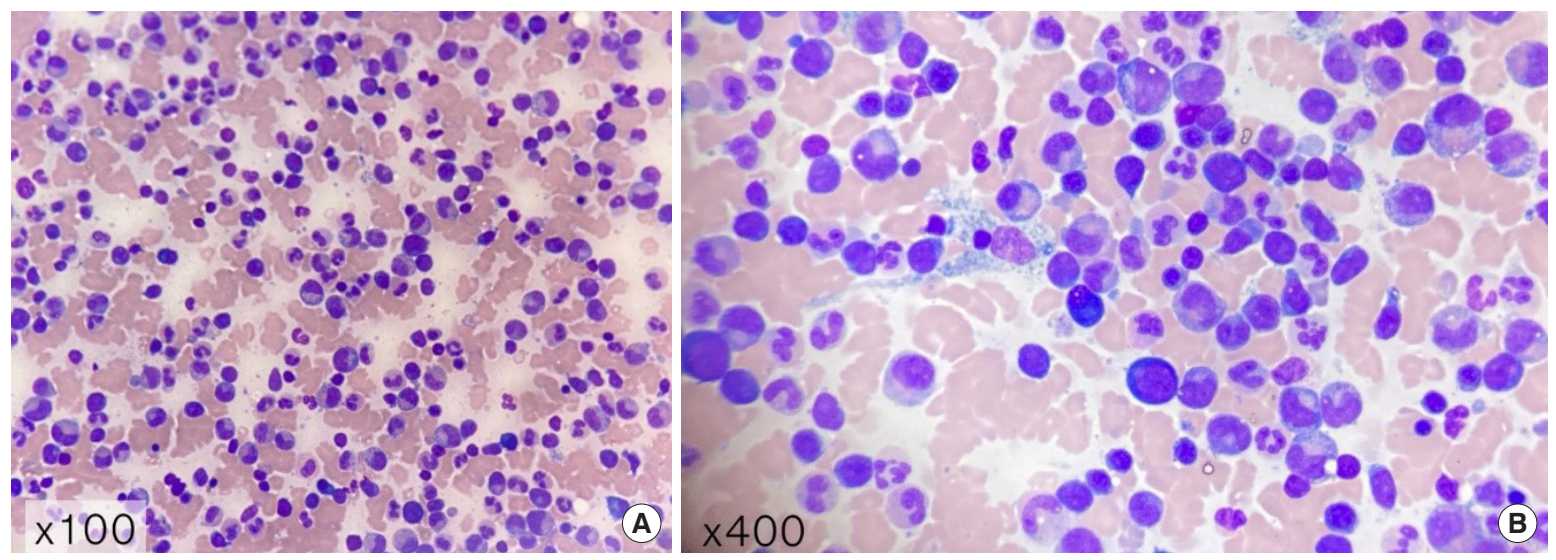

Fig. 5. Bone marrow biopsy findings. (A, B) Hypercellular (90\%) bone marrow section relative to the patient's age, increased myeloid series in proportion to the increased myeloblasts $(7.4 \%)$, normal erythroid series, and an adequate number of megakaryocytes were observed, suggesting myelodysplastic syndrome with excess blast-2 (Wright-Giemsa stain).

experienced persistent high fever. Considering the history of pancytopenia, the patient was sent for a re-consultation and further evaluation at the hemato-oncology department. A bone marrow biopsy was performed, and the patient was finally diagnosed with MDS (Fig. 5).

During the second hospitalization, the patient was managed 
only with conservative dressing and intravenous antibiotics. The patient was discharged with improvement in cellulitis and treated at the hemato-oncology department (Fig. 4C). The cause of the unexplained recurrent cellulitis in this case was MDS.

\section{Discussion}

MDS are clonal disorders of malignant hematopoietic stem cells characterized by dysplastic and ineffective hematopoiesis, resulting in a high risk of transformation to acute myeloid leukemia $[1,2]$. They occur most commonly in older adults $[3,4]$. MDS have been associated with benzene derivatives, radiation, tobacco, chemotherapy, and inherited genetic abnormalities $[2,5]$.

Patients with MDS may experience prolonged periods of fatigue or exertional dyspnea over 6-12 months, recurrent infections, petechiae, and other signs of bleeding. However, many patients are asymptomatic at diagnosis, and abnormalities in routine blood counts such as anemia, neutropenia, and thrombocytopenia are usually found first [6].

The diagnosis of MDS is based on a peripheral blood smear and bone marrow biopsy with a suggestive clinical context. Significant dysplasia in erythroid precursors, granulocytes, or megakaryocytes is observed on the blood smear or bone marrow examination [7]. Cytogenetic abnormalities can be the evidence of MDS in cases with no morphologic evidence of dysplasia.

The high risk of infection in MDS patients is well known and is considered a significant cause of morbidity and mortality $[2,8]$. Most infectious complications originate from bacteria, and cellulitis accounts for approximately $30 \%$ of infectious complications [8]. The infections are mainly attributed to the quantitative and qualitative defects in neutrophils. Guidelines for the treatment of infection in MDS patients have not yet been established. Some studies have shown that granulocyte colony-stimulating factor improves neutropenia in $30 \%$ to $70 \%$ of neutropenic MDS cases; however, its efficacy in preventing infection has not been proven [8]. Meanwhile, antibacterial prophylaxis may increase bacterial resistance, but the benefits and risks of prophylactic antimicrobials are not confirmed. Use of antibacterial prophylaxis is believed to be reasonable in patients who start therapy with demethylating agents and in those with a neutrophil count under $500 \times 10^{9} / \mathrm{L}$ [8]. Patients with MDS should be aware of the risk of infection. In case of febrile neutropenia, patients should be admitted with immediate tests, including blood cultures. Immediate broad-spectrum empirical antibiotics are warranted in such patients, and the clinical presentation should be considered when choosing the antibiotics.

This case is significant as our patient had no infections other than the recurrent cellulitis and his routine blood counts were at the limit of the normal ranges. While the high risk of infection in MDS patients is well-characterized, diagnosing MDS as the underlying cause of recurrent cellulitis is challenging, especially since cellulitis has various causes and often recurs, and MDS themselves are rare diseases [4,9]. Since many patients with MDS are asymptomatic and the clinical manifestations are not specific, an abnormal routine blood count is important when suspecting MDS. The initial laboratory findings of this patient suggested pancytopenia; however, his blood count was at the limit of the normal range. Patients with borderline laboratory results are likely to be misdiagnosed. Indeed, the hemato-oncologist suspected that the mild neutropenia and thrombocytopenia was possibly due to inflammation. The history of intermittent hematochezia could explain his low hemoglobin level. Furthermore, the lack of other symptoms or infections mean that there was no evidence of immunodeficiency. Thus, the patient was thought to have an uncontrolled infection of the skin lesion.

In cases of recurrent cellulitis with findings suggesting pancytopenia, predisposing factors that could cause immunosuppression should be considered. Hematologic malignancies, including MDS or acute leukemia, can cause pancytopenia through bone marrow infiltration. The use of cytotoxic drugs, underlying autoimmune diseases, poor nutritional status, and viral infections such as human immunodeficiency virus (HIV) are all possible factors associated with bone marrow failure [10]. Diabetes mellitus is a common predisposing factor that can cause secondary immunodeficiency. The patient in this case had no medical history of cytotoxic drugs, no underlying diabetes mellitus, and no autoimmune diseases. Tests for viral infection, including HIV and hepatitis, showed negative results. There were no problems with his nutritional status. Through bone marrow biopsy, we found that his pancytopenia could be attributed to MDS.

Sweet syndrome is a disease that can mimic cellulitis in patients with MDS [11]. Differential diagnosis is necessary because Sweet syndrome responds well to systemic glucocorticoids. In this case, Sweet syndrome could not be histologically ruled out because neutrophilic dermatosis without clear evidence of leukocytoclastic vasculitis was seen. However, the 
skin lesion was far from the erythematous plaques seen in Sweet syndrome [12].

This case shows that recurrent cellulitis that is difficult to resolve may not simply be a case of uncontrolled infection. If the patient's laboratory findings suggest pancytopenia, the recurrent infection could be attributed to systemic reasons, such as MDS.

\section{Conflict of interest}

This work was supported by the research fund of Hanyang University (HY-2020). Otherwise, no potential conflict of interest relevant to this article was reported.

\section{ORCID iDs}

Jung Han Lim https://orcid.org/0000-0002-0202-3674

Sue Min Kim https://orcid.org/0000-0002-0894-5207

Seungyun Jee https://orcid.org/0000-0003-3895-817X

Seong Oh Park https://orcid.org/0000-0001-8990-0635

\section{References}

1. Walter MJ, Shen D, Ding L, et al. Clonal architecture of secondary acute myeloid leukemia. N Engl J Med 2012; 366:1090-8.

2. Toma A, Fenaux P, Dreyfus F, et al. Infections in myelodysplastic syndromes. Haematologica 2012;97:1459-70.

3. Foran JM, Shammo JM. Clinical presentation, diagnosis, and prognosis of myelodysplastic syndromes. Am J Med
2012;125(7 Suppl):S6-13.

4. Williamson PJ, Kruger AR, Reynolds PJ, et al. Establishing the incidence of myelodysplastic syndrome. Br J Haematol 1994;87:743-5.

5. Schnatter AR, Glass DC, Tang G, et al. Myelodysplastic syndrome and benzene exposure among petroleum workers: an international pooled analysis. J Natl Cancer Inst 2012;104:1724-37.

6. Hofmann WK, Koeffler HP. Myelodysplastic syndrome. Annu Rev Med 2005;56:1-16.

7. Mufti GJ. Pathobiology, classification, and diagnosis of myelodysplastic syndrome. Best Pract Res Clin Haematol 2004;17:543-57.

8. Leone G, Pagano L. Infections in myelodysplastic syndrome in relation to stage and therapy. Mediterr J Hematol Infect Dis 2018;10:e2018039.

9. Raff AB, Kroshinsky D. Cellulitis: a review. JAMA 2016; 316:325-37.

10. Gnanaraj J, Parnes A, Francis CW, et al. Approach to pancytopenia: diagnostic algorithm for clinical hematologists. Blood Rev 2018;32:361-7.

11. Miura T, Misa K, Yamamoto T. Subcutaneous Sweet syndrome mimicking cellulitis in a patient with myelodysplastic syndrome and subsequent secondary pulmonary alveolar proteinosis. Clin Exp Dermatol 2020;45:763-4.

12. Rochet NM, Chavan RN, Cappel MA, et al. Sweet syndrome: clinical presentation, associations, and response to treatment in 77 patients. J Am Acad Dermatol 2013;69: 557-64. 